\title{
Granular Cell Tumor of the Breast: A Case Report
}

Garrett Fiscus ${ }^{1}$, Naomi Wiens ${ }^{2}$, Brynn Wolff ${ }^{2}$ and Yanping $\mathrm{Li}^{3}$

${ }^{1}$ Lake Erie College of Osteopathic Medicine, 1858 W Grandview BIvd, Erie, Pennsylvania 16509, USA

2UPMC Pinnacle Breast Care Center, UMPC Pinnacle Breast Care Center, 4300 Londonderry Rd Suite 202, Harrisburg, PA 17109, USA

${ }^{3}$ Pathology Associates of Central Pennsylvania, UMPC Pinnacle Harrisburg Hospital, 100 S. 2nd St, Harrisburg, PA 17101, USA

*Corresponding author: Garrett Fiscus, Lake Erie College of Osteopathic Medicine, 1858 W Grandview Blvd, Erie, Pennsylvania 16509, USA, Tel: +18147068168; Email: gfiscus34879@med.lecom.edu

Received Date: Dec 27, 2018; Accepted Date: Jan 14, 2019; Published Date: Jan 23, 2019

Copyright: (c) 2019 Fiscus G, et al. This is an open-access article distributed under the terms of the Creative Commons Attribution License, which permits unrestricted use, distribution, and reproduction in any medium, provided the original author and source are credited.

\begin{abstract}
Granular cell tumor (GCT) is a rare benign soft tissue neoplasm of neural origin occurring virtually any body site, breast localization accounting for between $5 \%$ and $15 \%$ of all cases. Clinically and radiologically, GCTs display similar signs of malignancy with carcinoma, hindering the ability of a confident diagnosis. GCTs show a distinctive histological pattern of granular eosinophilic cytoplasm with S100 positivity.
\end{abstract}

We report the case of a 67-year-old woman who presented with a painful palpable mass in her left axillary tail for over 30 years. Mammography and ultrasound revealed a 15-mm, ill-defined hypoechoic area with shadowing and vascularity within her left axillary tail. Excisional biopsy was performed and postoperative histological examination with immunohistochemical staining confirmed the diagnosis of a granular cell tumor.

This case is of clinical interest due to the atypical examination findings of location and chronicity, in addition to the rarity of GCT within the axillary tail of the breast. Proper knowledge is needed for a broad differential for breast tumors.

Keywords: Breast; Granular cell tumor; Peripheral nerve sheath tumor; S100 protein; Breast surgery

\section{Introduction}

Granular cell tumor is a rare benign soft tissue neoplasm initially believed to arise from mesenchymal cells (e.g. fibroblasts, myocytes) and Schwann cells due to the tumor's positivity to S100 protein $[1,2]$. However, the most recent accepted theory states that the origin is of nerve sheath [2-4]. Most incidences are benign, however, malignant GCTs have been documented in approximately $1-2 \%$ of cases $[3,5-7]$ GCTs arise at virtually any body site, mainly affecting the head neck region, skin or subcutaneous tissues. It was first identified by Weber et al. [8] in the tongue in 1854, and then Abrikossoff et al. [9] were the first to document its breast involvement in 1931. Localization to the breast is very rare, accounting for between $5 \%$ to $15 \%$ of all GCT cases $[10,11]$. It is imperative to differentiate GCTs from breast carcinoma as GCTs may simulate carcinoma diagnostically on mammography and ultrasound, as well as clinically with a nontender palpable breast mass [12]. We report on our findings in a patient with an atypical presentation of GCT in a rare location, within her left axillary tail.

\section{Case Report}

A 67-year old postmenopausal woman first noted a non-tender fixed mass in her upper outer left breast over 30 years ago on selfexamination. It was evaluated at that time with diagnostic breast imaging and clinical examination, but deemed likely nonmalignant. She underwent several years of surveillance with no interval change, never having excisional or core needle biopsy of the lesion performed.
The patient's demographics were significant for a healthy African American gravida 1 para 1 female with menarche at age 13 and first living birth at age 29 . The patient had a negative screening colonoscopy less than 5 years from surgical consultation, no prior operations, took a multivitamin only, and there was not any family history of breast or ovarian tumors. Both her brother and sister were treated for colon cancer. She denied any hormonal use, alcohol or drug use. She was a former smoker with a 20-pack year history and a BMI of 24.8.

In November 2017, an area of architectural irregularity in the left breast axillary tail versus the left axilla was noted on screening mammography, BIRADS 0 (Figure 1). Diagnostic ultrasound found a $15-\mathrm{mm}$, ill-defined hypoechoic area with shadowing and vascularity within her left axillary tail without surrounding adenopathy, BIRADS 4 (Figures 2 and 3). A core needle biopsy in December 2017 revealed fibroadipose tissue, discordant with imaging. Excisional biopsy was recommended.

Single incision excisional biopsy with sentinel lymph node biopsy using methylene blue dye and technetium-99m sulfur colloid radiotracer was performed 4 weeks after the core needle biopsy. The operative dictation noted the mass was firm and suspicious for malignancy. Microscopically, a benign neoplastic tumor was observed. The tumor was composed of cords of polygonal bland cells with welldefined cells borders, abundant eosinophilic granular cytoplasm and small round nuclei permeating through the collagenous stroma, consistent with a GCT. No features of malignancy such as tumor cell spindling, necrosis, mitosis, or nuclear atypia were identified. The immunohistochemical analysis showed the following results: strongly positive staining for S-100 protein, CD68, and vimentin; and negative 
staining for AE1/3, SMA, and CD34 (Figure 4). No malignancy was identified in the surgical specimen or two lymph nodes removed.

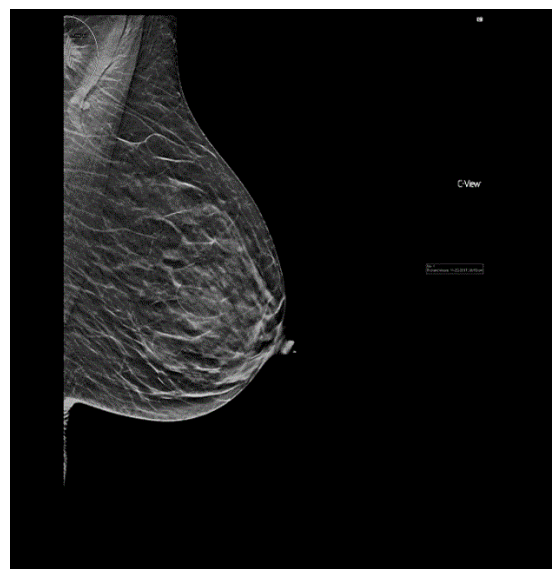

Figure 1: Screening mammogram of left breast, medial lateral oblique view, with radiographic circle around a peripheral spiculated lesion (November 21, 2017).

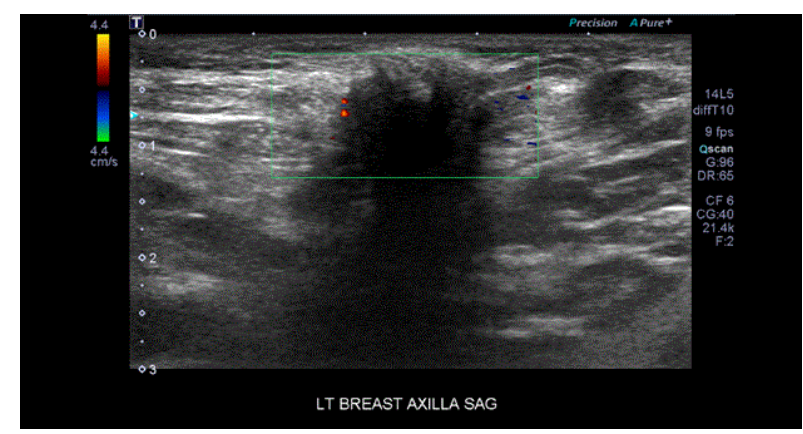

Figure 2: Ultrasound of left breast, sagittal view, with vascularity noted within a hypoechoic, irregular lesion with shadowing (December 6, 2017).

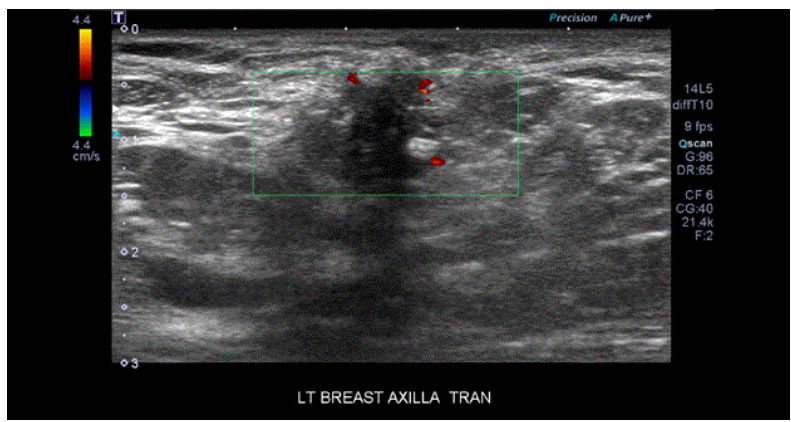

Figure 3: Ultrasound of left breast, transverse view, with vascularity noted within a hypoechoic, irregular lesion with shadowing (December 6, 2017).

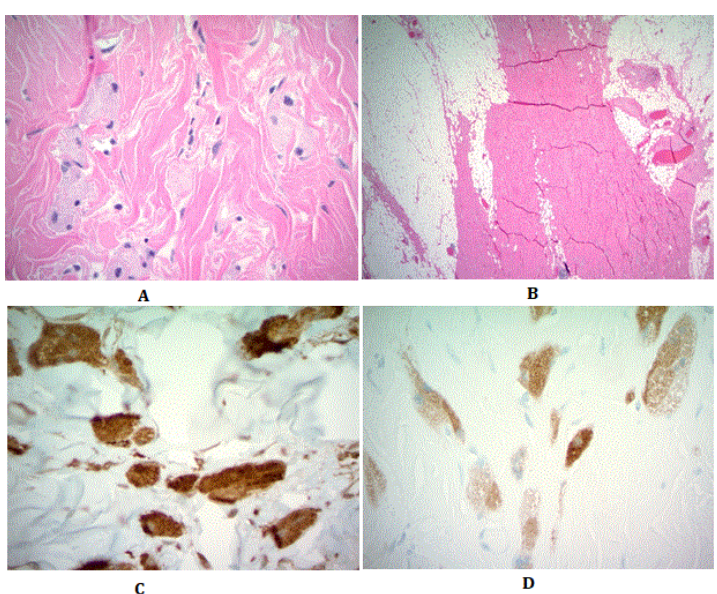

Figure 4: (A) High and low power view of the tumor, showing cords of tumor cells with abudant granular cytoplasm and (B) spiculated appearance at low power with collagen bands dissecting through the adipose tissue. (C) The tumor cells are strongly positive for CD68 and (D) S100.

Post operatively the patient developed swelling and discomfort in the left axilla consistent with seroma. She required aspiration twice. She continued to follow up for 4 weeks postoperatively and did not require further therapy. She was scheduled to continue with yearly mammography.

\section{Discussion}

GCTs remain a rare, typically benign neoplastic tumor of peripheral nervous tissue origin, occurring in adults with a female preponderance. Breast involvement has been described in $5-15 \%$ of reported cases, with approximately $1-2 \%$ of cases having malignant potential (i.e. dimpling, ulcerations, fascial/muscular affixations) [3,5-7]. Most breast GCTs present as a spiculated ill-defined, firm painless mass, typically arising from the intra-lobular breast stroma within the upper inner quadrant of the breast, contrary to the axillary tail presentation noted in our patient [1-3]. The appearance of a GCT in axillary region is extremely rare as there have been two documented reports of axillary involvement. Aoyama et al. [3] and Hammas et al. [11] reported a total of two, out of seven total cases of GCTs, in which there was axillary region involvement. They exhibited no laterality preference.

Clinical examination, as well as diagnostic imaging for GCTs are nonspecific, allowing it to be easily misdiagnosed as malignant carcinoma. Mammography shows wide range of appearance from small, well-circumscribed lesions to spiculated masses. Microcalcifications are not a routine feature seen in GCTs. Ultrasound evaluation usually demonstrates a solid hypoechoic mass with posterior shadowing and increased vascularity, as was seen in our case. Because of the variable presentation of GCTs seen on diagnostic imaging, it is imperative to get a biopsy of the lesion to facilitate proper diagnosis.

On pathological examination, GCTs have a distinctive granular eosinophilic cytoplasm associated with typical nuclei. The cells are generally uniform, large, polygonal, or spindle shaped with abundant 
granular-appearing cytoplasm, hence where the tumor gets its name. This distinguishing characteristic is due to the abundance of eosinophilic granules within the cytoplasm of the cells, from the accumulation of cell membrane infoldings, similar to myelin formation of axons. Subsequently, lysosomes appear to phagocytose these infoldings, producing the typical granular pattern seen in a GCT [13]. The most recent accepted theory of histogenesis states that the origin is of nerve sheath or of neuroectodermal origin due to the presence of S100 protein, which is typically expressed by these cells, supporting the derivation of Schwann cells. Immunohistochemically, they are strongly positive for PAS, CD68, S100 protein, calretinin, and neuron specific endolase, with some positivity to the $\alpha$-subunit of inhibin in GCTs and negativity to cytokeratins, estrogen and progesterone. In our presented case, analysis showed strongly positive staining for S100 protein, CD68, and vimentin; with negative staining for AE1/3, SMA, and CD34.

Early detection combined with wide local excision of the tumor remains the treatment of choice. Wide resection with adequate margins is necessary since the tumor has no capsule, allowing for local invasion either lymphatically or hematogenously. The suspicious findings on diagnostic mammogram, ultrasound and intra-operative examination support the role of lymph node biopsy in the presented case. However, a concordant core needle biopsy may have led the surgeon to forgo sentinel lymph node biopsy and decrease the patient's risk of developing a post-operative seroma. The presented case was treated accordingly with expected follow up and has had no signs or symptoms of early local recurrence to date.

\section{Conclusion}

This case illustrates that proper knowledge of broad differentials, including the possibility of a GCT diagnosis, is needed to make a reliable diagnosis and treatment plan. Although GCTs are rare, and occur in less than $15 \%$ of breast tumors, GCTs should be in the differential diagnosis of both benign and malignant breast tumors. Because the examination findings are nonspecific, it is imperative to obtain a tissue biopsy as early as possible, as the main striking element of GCTs is histologic. Protein S100 positivity, in addition to CD68 expression, has been a useful diagnostic marker for GCTs. The case discussed is indeed an uncommon presentation of a rare tumor, and this knowledge may help facilitate clinicians treat suspicious breast lesions appropriately in the ever advancing field of breast oncology.

\section{Disclosure of Interest}

The authors declare that they have no conflicts of interest in the write-up of this article.

\section{References}

1. Filipovski V, Banev S, Janevska V, Dukova B (2009) Granular cell tumor of the breast: a case report and review of literature. Cases J 2: 8551.

2. Pohlodek K, Jáni P, Mečlarova I (2018) Granular cell tumor in axillary region: A rare entity. Mol Clin Onco 8: 579-581.

3. Aoyama K, Kamio T, Hirano A, Seshimo A, Kameoka S (2012) Granular cell tumors: a report of six cases. World J Surg Oncol 10: 204.

4. Pergel A, Yucel AF, Karaca AS, Aydin I, Sahin DA, et al. (2011) A therapeutic and diagnostic dilemma: granular cell tumor of the breast. Case Rep Med 2011: 972168.

5. Khansur T, Balducci L, Tavassoli M (1987) Granular cell tumor. Clinical spectrum of the benign and malignant entity. Cancer 60: 220-222.

6. Fanburg-Smith JC, Meis-Kindblom JM, Fante R, Kindblom LG (1998) Malignant granular cell tumor of soft tissue: diagnostic criteria and clinicopathologic correlation. Am J Surg Pathol 22: 779-794.

7. Sonobe H, Iwata J, Furihata M, Moriki T, Ohtsuki Y (1998) Malignant granular cell tumor: report of a case and review and the literature. Pathol Res Pract 194: 507-513.

8. Weber CO, Virchow R (1854) Anatomical examination of a hypertrophic tongue together with remarks on the formation of striated muscle fibers. Archive for pathological anatomy and physiology and for clinical medicine 7: 115-125.

9. Abrikossoff AI (1931) Weitere Untersuchungen über Myoblastenmyome. Virchows Arch Pathol Anat Physiol Klin Med 280: 723-740.

10. Brown AC, Audisio RA, Regitnig P (2011) Granular cell tumour of the breast. Surg Oncol 20: 97-105.

11. Hammas N, El Fatemi H, Jayi S, Hafid I, Fikri G, et al. (2014) Granular cell tumor of the breast: a case report. J Med Case Rep 8: 465.

12. Tran TA, Kallakury BV, Carter J, Wolf BC, Ross JS (1997) Coexistence of granular cell tumor and ipsilateral infiltrating ductal carcinoma of the breast. South Med J 90: 1149-1151.

13. Mittal KR, True LD (1988) Origin of granules in granular cell tumor. Intracellular myelin formation with autodigestion. Arch Pathol Lab Med 112: 302-303. 